DOT/FAA/AM-02/1

Office of Aerospace Medicine Washington, DC 20591

\section{An Information Tool for Planning in Air Traffic Control}

\author{
Scott D. Gronlund \\ John M. Canning \\ Peter M. Moertl \\ Joakim Johansson \\ University of Oklahoma \\ Department of Psychology \\ Norman, OK 73019 \\ Michael R. P. Dougherty \\ Department of Psychology \\ University of Maryland \\ College Park, MD 20742 \\ Scott H. Mills \\ SBC Technology Resources, Inc. \\ Austin, TX 78759
}

January 2002

Final Report

This document is available to the public through the National Technical Information Service, Springfield, VA 22161.

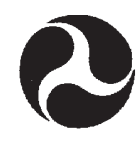

U.S.Department of Transportation

Federal Aviation

Administration 


\section{$\mathrm{N} O \mathrm{~T}$ I C E}

This document is disseminated under the sponsorship of the U.S. Department of Transportation in the interest of information exchange. The United States Government assumes no liability for the contents thereof. 
Technical Report Documentation Page

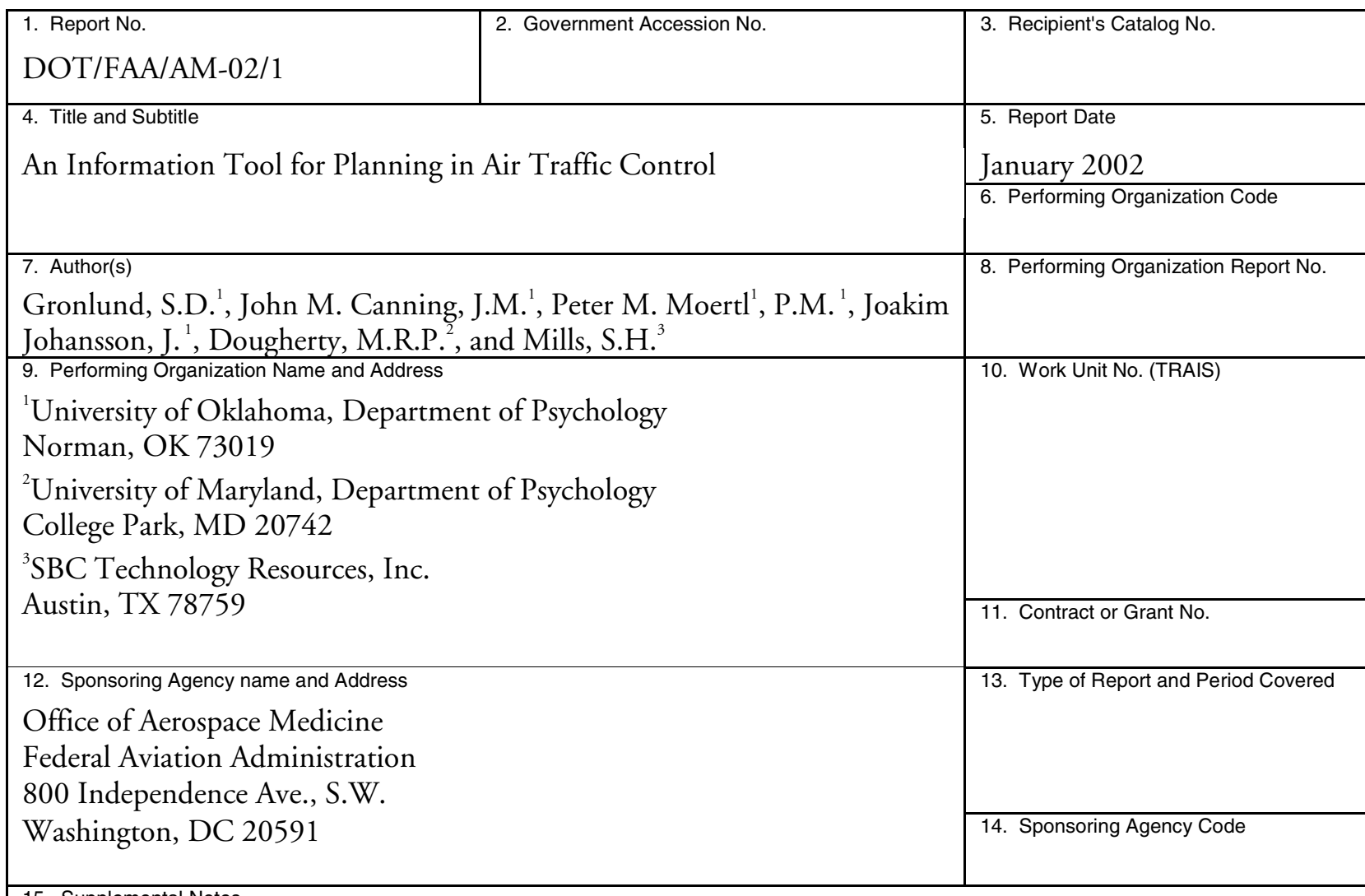

15. Supplemental Notes

This work was accomplished under grant \# 97-G-037, Approved Subtask \# AM-A-00-HRR-516.

16. Abstract

An investigation of how en route air traffic controllers solved sequencing problems using paper flight progress strips led to the development of a new software interface to aid planning. The primary feature of the new interface involved replacing the strips with virtual tokens that were dynamically linked to their radar targets. In addition, an automated tool was developed that provided a tentative sequencing of the tokens. The current experiment evaluated the automated sequencing tool. Utilization of the tool facilitated the development of the controllers' plans and reduced the workload of the tacticians who implemented the plan.

\begin{tabular}{|l|l|l|l|}
\hline $\begin{array}{l}\text { 17. Key Words } \\
\text { Air Traffic Control, Automation, Interface, Planning }\end{array}$ & $\begin{array}{l}\text { 18. Distribution Statement } \\
\text { Document is available to the public through the } \\
\text { National Technical Information Service; } \\
\text { Springfield, VA 22161 }\end{array}$ \\
\hline $\begin{array}{c}\text { 19. Security Classif. (of this report) } \\
\text { Unclassified }\end{array}$ & $\begin{array}{l}\text { 20. Security Classif. (of this page) } \\
\text { Unclassified }\end{array}$ & $\begin{array}{l}\text { 21. No. of Pages } \\
14\end{array}$ & 22. Price \\
\hline
\end{tabular}





\section{Acknowledgments}

We gratefully acknowledge the support of FAA Grant No. 97-G-037. We are indebted to Ron Andrei (AMA-510) and Paul DeBenedittis (AMA-510) for their help on this project. This manuscript was improved by the comments of Carol Manning and Scott Shappell of the FAA Civil Aerospace Medical Institute (AAM-510). We also thank Jorge Mendoza for his statistical counsel. 



\section{An Information Organization Tool for Planning in Air Traffic Control}

Planning is fundamental to the successful completion of many complex, dynamic tasks. For example, Miller, Copeland, Heaton, and McCloskey (1998) found planning to be important for successful military operations (see also Pew \& Mavor, 1998); Xiao, Milgram, and Doyle (1997) found it to be an important aspect of anesthesiologists' preparation for surgery. Although air traffic control has been characterized as largely involving shorter-term tactical decision making (Durso \& Gronlund, 1999; Hutton, Olszewski, Thordsen, \& Kaempf, 1997), planning is also an important contributor (e.g., David, 1997; Dougherty, Gronlund, Durso, Canning, \& Mills, 1999). Roughly speaking, by tactics we mean decisions that relate to the current moment and involve the separation of (usually) pairs of aircraft; planning decisions occur further in advance than tactics and involve the consideration of a larger number of aircraft.

An examination of planning in air traffic control is timely, given future concepts being proposed. For instance, there have been discussions regarding the creation of a strategic controller position (N. Lawson \& K. Thompson, personal communication, Dec. 15, 1997; see also Vivona, Ballin, Green, Bach, \& McNally, 1996). The proposal provides for one person who would be responsible for a multiple-sector airspace, making decisions about traffic in that airspace, and delegating responsibility for tactical decisions to sector-level controllers. One goal of our project was to develop possible interface tools for a strategic controller position.

Before developing new interface tools to aid planning or to support a strategic controller position, it is important to better understand how controllers use their current tools to develop plans. As pointed out by David (1997, p. 13), this is nontrivial because it "appears to be a cognitive task almost invisible to the outside observer. " In addition, tactics and planning are normally confounded in air traffic control because both types of decision-making often lie within the same head, even when a team has responsibility for a sector of airspace.
Dougherty et al. (1999) solved both problems by assigning the role of the planner and the role of the tactician, the implementer of the plan, to two different people and requiring that the planner verbalize the plan for the tactician to implement. The experiment began with instructions that delineated the roles of the tactician, the subject matter expert in the experiment, and the planner who served as the participant. The tactician's job was to maintain separation between aircraft; that is, he or she made whatever altitude, speed, and heading changes were necessary to maintain separation. The planner's job was to give the tactician a plan for managing the flow of traffic in the sector (a volume of airspace). The planners conveyed their plan to the tactician by verbalizing it. The planners sat beside the radar (the tactician sat in front of it) and in front of the flight progress strips. ${ }^{1}$

Twelve en route air traffic controllers serving as instructors at the FAA Academy in Oklahoma City completed two different types of scenarios. In one scenario, the aircraft flew standard routes (so-called highways in the sky) in which pairs of aircraft would only intersect at a restricted number of points along the route. The primary problem to be solved involved sequencing aircraft into Dallas/Fort Worth. For the other scenario, aircraft flew direct routes through the sector, which meant that pairs of aircraft could intersect at any point in the sector. In this scenario, aircraft were en route to many different destinations. The scenarios were initially presented in a paused mode that allowed the planner the necessary time to formulate a plan. In a pilot study, we found that the planner would immediately fall into a tactical mode if the problem was active when first viewed.

The direct route scenario required more decisions relating to the separation of pairs of aircraft rather than decisions about larger groups of aircraft. However, the latter were much more frequent in the sequencing scenario, and because these problems are more the province of a strategic controller, we focused on sequencing problems in the interface development effort to follow. In the sequencing scenario, planners

\footnotetext{
${ }^{1}$ Flight progress strips for each aircraft were stacked vertically in a strip bay adjacent to the radar display. Flight strips are $20 \times 3 \mathrm{~cm}$ rectangular paper strips. They have 31 fields of information, including the call sign, aircraft type, requested altitude, requested speed, route of flight, etc. The controllers mark on these strips to update this information. In the field, paper strips are important, in part, because they can function as a manual backup in case the radar and computers fail.
} 
made greater use of the flight progress strips. Ten of the 12 planners built their sequence of aircraft using only the strips (one planner used the strips and the radar, the other used only the radar). In addition, a greater proportion of the strips were marked in the sequencing scenario (.45 vs. .24 , all $p$ 's $<.05$ unless otherwise indicated), and more strips were moved at the beginning of the problem (5.7 vs. 1.5). This occurred despite the fact that the planners were instructed that the tactician would not use the strips and that they were only for the planner's benefit.

Several sub-tasks performed by the controllers in Dougherty et al. (1999) related to their decisionmaking in the sequencing scenario. The two most important tasks were classifying the aircraft into groups and sequencing the aircraft within each group. The classifications were needed to identify the set of aircraft that would be affected by a particular constraint such as "maintain 10 miles in trail between aircraft on jetroute 107." The sequences were needed to determine in which order aircraft would pass a common ground point fix. These fixes were related to the route or destination of the aircraft (e.g., a hand-off location to the next sector). To perform their classification and sequencing sub-tasks, planners needed to: 1) find current flight status information (position, altitude, and speed), and 2) find current flight plan information (destination and intermediate points). All of the planners had grouped their flight progress strips by aircraft destination and then ordered them within each group by arrival time at a particular fix. The strips served as tokens for the aircraft, and the stacking order of the tokens made the sequence order explicit.

To enhance the functionality of the paper flight strips, a software interface was designed (see Canning, Johansson, Gronlund, Dougherty, \& Mills, 1999). Two screens were created for the planner: a radar view and a flight organizer (see Figures 1 and 2). The planner's radar display looked much like the radar portion of the Display System Replacement (DSR) screens used by the FAA. However, the radar display was enhanced to allow for the color coding, marking, and highlighting of the aircraft in coordination with the flight organizer display. Classifying and sequencing took place on the flight organizer display. Each aircraft was represented by a small rectangle called an aircraft token. Sets of aircraft tokens were placed into containers called queuing blocks which represented the groups of classified aircraft. All aircraft tokens placed in a queuing block were displayed in the same color. The corresponding aircraft symbol on the radar view appeared in that same color. Therefore, the classification was encoded using color on both dis- plays. Planners created and positioned as many queuing blocks as they wished on the flight organizer screen and selected a fix for each one. Time or distance to reach the fix (at the option of the planner) was displayed to the right of each aircraft token. An automated sequencing tool allowed the aircraft tokens to be automatically reordered so that the lowest token was the one closest to that block's fix, and the other tokens were in ascending order.

The automated sequencing tool did not create an optimized ordering of tokens (as would a planning aid like CTAS, Vivona et al., 1996; or URET, Brudnicki \& McFarland, 1997). Rather, it ordered the tokens by the time or distance to reach the chosen fix, based on the aircraft's current position, heading, and speed. It provided a first attempt at a sequence order that could then be fine-tuned by the planner. This was in keeping with a philosophy of automation that keeps the operator in the loop (human-centered automation, e.g., Billings, 1996). According to this philosophy, new tools should allow more traffic to be handled and better decisions to be made but do so not by outsourcing the cognitive ability of the operator to the tools. Rather, through restructuring the information and speeding access to critical information, an operator's cognitive abilities can be enhanced. For a similar philosophy applied to air traffic control see the PHARE Highly Interactive Problem Solver developed for Eurocontrol (Meckoff \& Gibbs, 1994).

Moertl et al.. (2000) reported the results of an experiment that evaluated the new interface. The same 12 planners participated as in the first experiment. They received about two hours of training on the new interface prior to participating. They completed two sequencing problems: one using the flight organizer together with the radar, and one using the strips and the radar (the color-coding functionality of the radar was lost without the flight organizer). As before, planners conveyed their plan to the tactician by verbalizing it. Planner performance (rated by the planner and tactician and collapsed over the 20minute scenario) was judged to be superior in the flight organizer condition (6.1 vs. 5.2, 9-point scale, 9 being best), although the advantage was only marginal during the first 10-minutes. The flight organizer also resulted in a lower workload over the 20 -minute scenario for both the planner and the tactician $(50.4$ vs. 57.8 , measured by the average of the six indices of the NASA Task Load IndeX that includes mental demand, physical demand, temporal demand, frustration, effort, and the assessment of performance, which was reverse scored (Hart \& Staveland, 1998). Although participants had as much time as they needed 


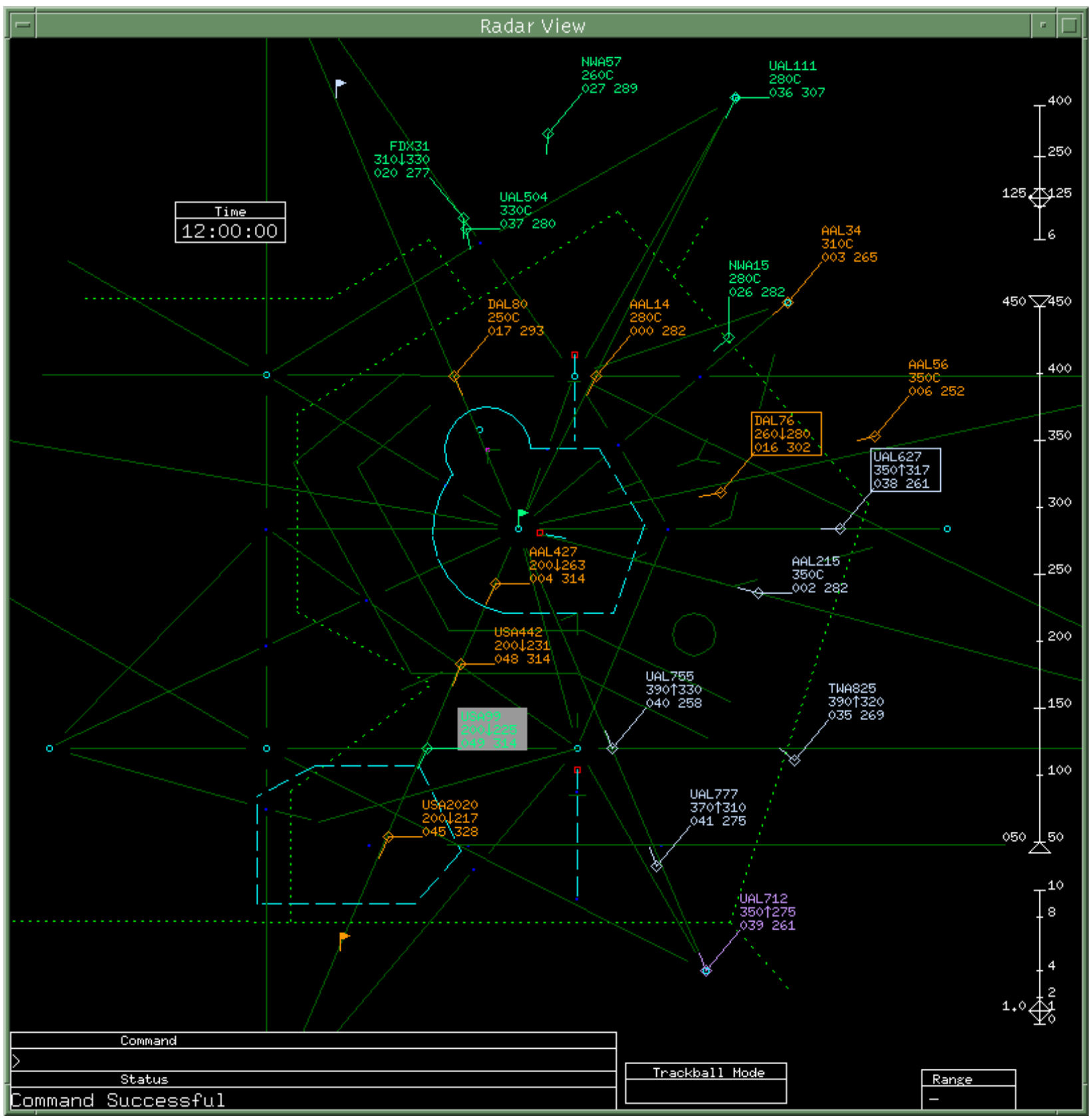

Figure 1. The Radar View: One of the two screens available to the planner. See details in the text and in Canning et al. (1999). 
to develop their plans, a plan was developed 5.4 minutes faster, on average, using the flight organizer than using the strips, despite only 2 hours of prior familiarization with the interface.

The new interface seemed to offer several advantages over the existing paper flight progress strip environment. The current experiment sought to identify the components of the new interface that were responsible for the benefit. In particular, the current experiment evaluated the effectiveness of the automated sequencing tool. In two of the three scenarios, the automated sequencing tool was fully functional. In the third scenario, the ability to use fixes with queuing blocks was disabled. Without a fix, the automated sequencing tool was of no use and planners would have to construct their own sequences of aircraft. The planners could still create queuing blocks, but they could not reorder the tokens within a block at the touch of a button.

One new feature was added to the interface for this experiment. The planner now had the capability to mark on the aircraft token the control actions they decided should be taken to achieve the planned sequence. Planners typed the appropriate letter and then clicked a token to indicate that a speed, vector, and/ or altitude control action was needed. The letters appeared within the token in the center of the bottom row (see Figure 2). The planner could mark more than one control action for a particular aircraft, and change it whenever necessary. The planner could also indicate that no control action was needed for a given aircraft (i.e., it would fall into place given its current speed, altitude, and heading). For example, the token for TWA825 in Figure 2 shows that speed should be used by the tactician to achieve the appropriate sequencing of this aircraft, while no action is indicated for UAL755, which meant that it should fall into place given its current speed, heading, and altitude. Also, rather than requiring that the planner verbally convey the plan to the tactician, the planner simply printed out a copy of the sequence order for the tactician that included the accompanying control actions. The tactician remained responsible for issuing the control actions to the pilots, and for issuing other control actions needed to maintain the necessary separation between aircraft.

Although the aforementioned annotation of aircraft tokens facilitated communication of the plan to the tactician, it also allowed for the introduction of a second independent variable into the experiment involving plan detail or specificity. Planners were required to create plans at one of two different levels of specificity. A partial sequence meant that the planner could sequence as many of the aircraft in a queuing block as they wished. A complete sequence meant that the planner had to create a more detailed plan by sequencing all the aircraft within a queuing block.

Planning aids like URET (Brudnicki \& McFarland, 1997) and CTAS (Vivona et al., 1996) allow controllers to make more detailed plans further into the future. On the surface, this seems like a good idea. But such plans might be particularly prone to cognitive rigidity (e.g., Taylor, Finnie, \& Hoy, 1997). Cognitive rigidity, a form of functional fixity, would occur when a planner stuck with an existing plan despite evidence (as judged by an outside observer) that the plan required modification. Cognitive rigidity can also be viewed as a form of sunk costs (Arkes \& Blumer, 1985). More time and effort devoted to developing a plan might make a planner more willing to maintain an existing plan, despite evidence that it should be modified. As a result, planning performance was predicted to be worse when a complete sequence was required.

\section{Method}

\section{Participants}

Five en route air traffic controllers participated as planners. All had participated in our prior interface experiment, which meant that they had experience using the flight organizer. None of the remaining participants from the prior experiment were available, and time and resources were unavailable to train additional participants on the interface. All participants were instructors at the FAA Academy and were familiar with the AeroCenter sector used in the experiment. All were full-performance level (FPL) controllers (i.e., certified to work a sector independently). They had been FPL controllers for an average of 20.5 years. They last worked in the field 3.8 years previously, on average. The tactician, our subject matter expert, was also an FPL controller.

\section{Materials}

The experiment was conducted at the Civil Aerospace Medical Institute in Oklahoma City. The planner sat at one end of a long room, and the tactician and two ghost pilots sat at the other end (the ghost pilots on either side of the tactician). The ghost pilots controlled the simulated aircraft based on the tactician's instructions. The planner and tactician communicated using an intercom system that simulated a radio. The tactician and the ghost pilots communicated over the tops of the cubicles that separated them. The tactician had a single screen on 


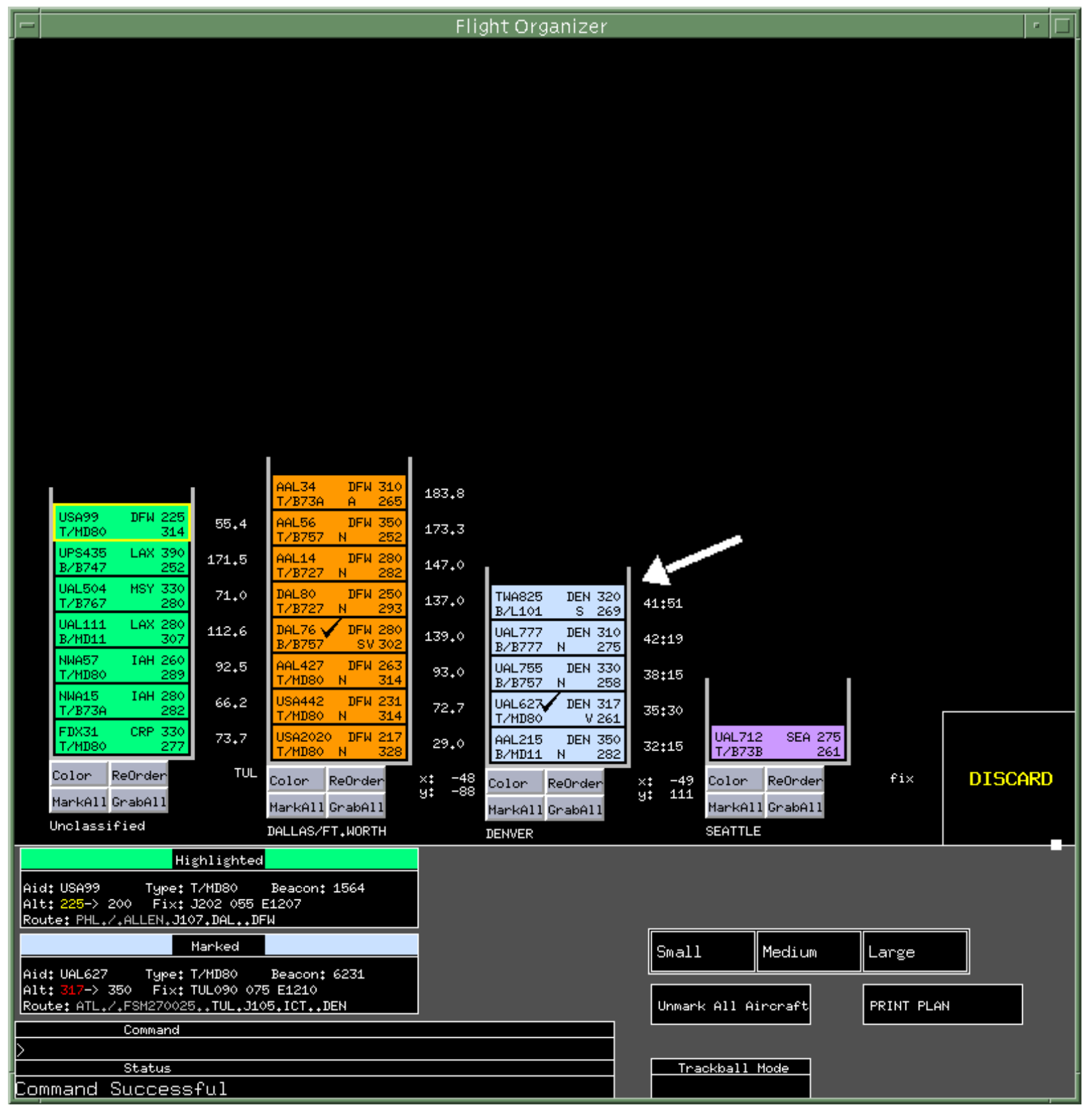

Figure 2. The Flight Organizer: One of the two screens available to the planner. See details in the text and in Canning et al. (1999). 
which a radar display was shown. The planner had the flight organizer and the radar display screens described above (see Figures 1 and 2).

Four scenarios were developed by the controller expert serving as the tactician. The scenarios were judged to exceed the workload level typically experienced in the field; there was an average of 36.5 aircraft to be handled during the 10-minute scenario. The primary problem to be solved involved sequencing aircraft into two different destination airports. These were called the primary sequences. Time constraints limited the experiment to completion of only three of the scenarios, although participants were rotated through all four.

\section{Procedure}

Each participant completed a 45-minute training session on the use of the flight organizer functions. This was followed one or two days later by a 45 -minute practice session with one of the trainers (graduate research assistants who were familiar with the interface). The experimental session began 2-3 days later.

There were three conditions in the experiment. In one, the planners created complete sequences for the two primary sequences in the scenario. Control actions needed to achieve the sequence had to be designated for all aircraft in the two primary sequences. In the other two conditions, planners created partial sequences for the two primary sequences. In other words, it was the planners' choice which aircraft had control actions designated and which aircraft had these decisions deferred. The automated sequencing tool was disabled for one of these two partial sequence problems. This meant that the planners had to construct their sequences by manually determining distances. The automated sequencing tool was enabled in the other partial condition and in the complete sequence conditions. In sum, the three conditions were: enabled-partial, disabled-partial, and enabledcomplete. See Table 1 for an overview of the requirements of the different conditions. The ordering of the conditions was randomized, as was the assignment of the four scenarios to the three conditions. ${ }^{2}$

The instructions began by delineating the roles of the tactician (the controller expert) and the planner (the participant). The tactician's job was to maintain separation between aircraft; he would make whatever altitude, speed, and heading changes were necessary to maintain separation. Only the tactician would communicate with the pilots. The planner's job was to sequence the aircraft in the two primary sequences. The planner was asked to indicate how the sequencing should be achieved by marking whether speed, altitude, heading or no control action was to be used to provide the necessary spacing and sequencing. The planner could print a copy of the plan for the tactician anytime the planner wished. The print-out contained an ordered list of the aircraft in the two primary sequences and the control actions designated by the planner to achieve the sequences.

The experiment began with the first scenario in the paused mode. The planners then configured the flight organizer to their preference. In particular, all the planners created at least two queuing blocks, one for each primary sequence. Although the planners were shown how the interface worked during training, we did not require that they use the interface in a particular way. Aircraft going to the appropriate destinations were then placed into the designated queuing blocks and sequenced either manually or by using the automated sequencing tool, depending on the condition.

After completing the plan, the planners pressed a button on the interface to print two hardcopies of the planned sequence. One copy was given to the tactician, who used this information to implement the plan. The other copy was given to the planner, who

\section{Table 1}

Experimental Conditions with Constraints

Aircraft Sequencing Control Action Marking Fix Status

Enabled-Partial

Disabled-Partial

Enabled-Complete

\begin{tabular}{|l|l|l|}
\hline Partial & Optional & Enabled \\
\hline Partial & Optional & Disabled \\
\hline Complete & Mandatory & Enabled \\
\hline
\end{tabular}

${ }^{2}$ Time constraints forced us to drop the disabled-complete condition. In a pilot study, plan preparation alone took over 20 minutes. 
was asked to circle aircraft considered to be in groups. Then, for each circled group of aircraft, the planners rated their confidence level that the aircraft in a group were in their final sequence order $(1=$ very certain to $5=$ very uncertain). Next, the planners rated their confidence level that the control actions marked were sufficient to establish the sequence order (again, $1=$ very certain to $5=$ very uncertain). Once the planners completed this process, the scenario was started and the tactician began implementing the plan. The planners were instructed to modify their plan as necessary and to print out the revised plan for the tactician whenever they wished.

The scenario was stopped after 10 minutes, and the tactician completed the NASA-TLX (Hart \& Staveland, 1998), which assessed mental demand, physical demand, temporal demand, frustration, and effort during the preceding 10 minutes. The NASATLX assessment of performance was excluded and replaced by having the planner and the tactician independently answer five questions related to the planner's plan: the planner's involvement in the tactics, quality of the initial plan, quality of revisions, planning effectiveness, and strategic awareness. A 10minute break followed. After finishing the three scenarios, the planner completed a questionnaire that collected biographical information. The participants were then debriefed and released. An experimental session took approximately 2.5 hours.

\section{Results}

The tactician's TLX workload differed significantly across conditions $(F(2,3)=15.82, p=.026$, see Table 2 ), according to Hotelling's (1931) $T^{2}$ test. Hotelling's $T^{2}$ test was preferred to a one-way ANOVA because it does makes no assumptions about the variance/covariance matrix or assumptions about the sphericity of the data. In addition, Marcucci (1986) showed that the $T^{2}$ test was a more powerful test when epsilon departs appreciably from 1.0 (the Greenhouse-Geisser epsilon was .585). The tactician's workload was significantly less in the enabled-partial condition than in the disabled-partial condition. This comparison revealed that the tactician's workload was reduced when the planner had access to the automated sequencing tool.

The tactician and the planner independently rated the quality of the planner's plan. The ratings were combined in Table 2 and in the following analysis. Although the plan was judged best in the enabledpartial condition and worst in the disabled-partial condition, consistent with the tactician's reduced workload when the planner used the automated sequencing tool, there were no significant differences across conditions $(F(2,3)=1.87, p=.3$, according to Hotelling's $T^{2}$ test). Although the lack of a significant difference could be a power problem, note that we had enough power to detect a change in the tacticians' TLX ratings.

\section{Table 2}

Tactician's Subjective Workload and Rating of Plan Quality for the Three Experimental Conditions (Standard Deviations Given in Parentheses).

\begin{tabular}{l|c|c|c|}
\multicolumn{1}{c}{} & \multicolumn{1}{c}{ Enabled-Complete } & \multicolumn{1}{c}{ Enabled-Partial } & Disabled-Partial \\
\cline { 2 - 4 } & 51.0 & 44.9 & 63.6 \\
Tactician's TLX & $(7.08)$ & $(14.27)$ & $(17.39)$ \\
\cline { 2 - 4 } $\begin{array}{l}\text { Rating of planner's } \\
\text { plan quality }\end{array}$ & 5.25 & 5.55 & 4.98 \\
& $(0.97)$ & $(1.35)$ & $(0.89)$ \\
\hline
\end{tabular}

\footnotetext{
${ }^{a}$ Averaged across the five workload subscales, performance excluded. Scale ranges from 0-100 with 100 indicating maximum workload.

${ }^{\mathrm{b}}$ Averaged across initial plan quality, quality of revisions, strategic awareness, and strategic planning. Scale ranges from 1 to 9 , with 9 indicating superior planning. These data represent the average of the tactician's and the planners' ratings.
} 
The lack of a difference in plan quality was probably because the planners had as much time as they wished to develop their plans. Therefore, an indication of the effectiveness of the automated tool might not manifest itself in an increased rating of plan quality but instead in a reduced time to develop the plan. In other words, as is often the case in cognitive psychology, a difference in processing efficiency can be observed in either the accuracy or the speed, depending on the parameters of the situation. For example, a difference in processing efficiency between students completing their first year versus their fifth year of study of a foreign language could result in a longer time for the first-year student to translate a passage, although they may eventually be able to translate it as accurately as the fifth-year student. However, if we give the first-year and the fifth-year students the same amount of time, less than what is needed by the first-year students, the time spent translating would not differ (by definition), but the difference in processing efficiency would result in reduced accuracy for the first-year students.

Use of the automated sequencing tool did reduce the time needed to complete plan development. Planners created their initial plan an average of 3.75 minutes more quickly when the automated sequencing tool was enabled than when it was disabled (enabled-partial 541 seconds, $s=180.9$ vs. disabled-partial 766 seconds, $s=275.8$ ). Although this difference was of marginal statistical significance (by a one-tailed test, $t(4)=1.93, p=.063)$, an improvement in plan development time of this size is of great practical significance to the controller.

Of primary interest in assessing the impact of plan specificity was the comparison of the enabled-complete to the enabled-partial condition. This manipulation resulted in control actions being indicated for $94 \%$ of the aircraft in the two primary sequences in the complete condition but only $65.5 \%$ of the aircraft in the two partial conditions. The tactician's workload was greater in the enabled-complete condition relative to the enabled-partial condition, but not significantly so. Although plan quality was judged worse when the planner created a more detailed plan in the enabled-complete condition, as was discussed above, the difference was not significant during the initial 10 minutes of the scenario. The support for the disadvantages of increased plan specificity was much weaker than the support for the advantages of the automated sequencing tool.
Small differences across conditions for the remaining results made the mean a sufficient summary. The planners circled an average of 5.2 aircraft as making up a group. This was larger than the group size of 2.7 aircraft reported by Means et al. (1988) in a task that involved circling recalled aircraft on a map. This difference may reflect the larger groups of aircraft considered by controllers in a planning role versus their more typical role. However, it may also reflect differences in aircraft density, instructions, questions asked, etc. Planners expressed high confidence in the sequence order they presented to tacticians ( $m=1.68$, 1 to 5 scale, $1=$ very certain). The majority of the control actions marked were speed (mean frequency of $m=13.3$ aircraft $)$ or vector $(m=9.4)$. Altitude changes were not important for solving the sequencing problems, $m=0.33$. No control actions were indicated for 5.8 aircraft on average. There was a marginal positive correlation between the number of speed and vector control actions $(r=.48, p<.07)$. Apparently, rather than a preference for planners to set-up their sequences using speed or vectors, the positive correlation suggested that planners tended to designate more of both types of control actions. However, a median split (all three conditions collapsed together) on the number of control actions showed that the tactician judged those planners who designated fewer control actions to have a better plan. Perhaps a planner with a better "big picture" of the sector knew the one control action that would solve a problem, while the planner with a poorer picture would have to solve the problem in pieces through a series of control action adjustments.

\section{Discussion}

The results of the experiment show consistent and beneficial effects of using the automated sequencing tool. The tactician's workload was decreased. Although plan quality was equivalent across conditions, plans were developed more rapidly using the automated sequencing tool. The results of Moertl et al. (2000) were similar. In that experiment, planners developed plans using the interface (including the automated sequencing tool) or the paper strips. Although plans were developed far more rapidly using the interface, there was no significant difference in plan quality during the initial 10 minutes of the scenario. Moertl et al. found superior planning performance in the second 10 minutes of the scenario when planners had to update and adapt their plans to the 
changing traffic situations. In the present experiment, the planners only completed 10 minutes per scenario. Perhaps differences in plan quality would have appeared if the scenario was run for 10 additional minutes.

The results of the experiment hint at a detriment to performance as a result of making more detailed plans. On the one hand, it seems like a good idea for controllers to use tools like URET and CTAS to look further into the future. On the other hand, having more detailed plans, plans that a controller might be more invested in, could result in greater cognitive rigidity and hence poorer performance. Additional studies that manipulate the level of detail of a plan and the degree of commitment to it should be conducted to verify that planning aids actually are beneficial. However, future attempts to examine this construct should consider other manipulations of plan specificity to include dependent variables better targeted at cognitive rigidity or, perhaps, situation awareness.

An examination of en route air traffic controller planning showed that an information organization tool could enhance the solving of sequencing problems. Prior research showed that the flight organizer was superior to the existing paper flight strip environment. In the current experiment, a component of the flight organizer, a sequencing tool that sorts aircraft tokens within a queuing block, was shown to decrease the workload of the tactician (who implemented the plan) and speed plan development. Dissecting a new interface is an important aspect of interface development as it can help determine what features of the interface are beneficial to performance and what aspects of performance they enhance. In the present experiment, we learned how the automated sequencing tool enhanced performance. Surprisingly, it was the tactician's workload, rather than the planner's, which benefited from the use of this tool. The tool did, however, reduce the time needed by planners to develop their initial plan. By dissecting an interface in this way, we can determine what features of the interface should be maintained and what those different features contribute to the overall performance of the system.

The flight organizer interface we designed for a future strategic controller position represents an alternative approach to air traffic control modernization. We first tried to understand how the controllers function in their current environment, and then tried to systematically enhance that functioning through targeted tools that kept the controller in the loop rather than outsourcing the planning process to software.

\section{References}

Arkes, H.R. \& Blumer, C. (1985). The psychology of sunk cost. Organizational Behavior \& Human Decision Processes, 35, 124-140.

Billings, C.E. (1996). Human-centered aviation automation: Principles and guidelines. NASA Technical Memorandum 110381, Moffett Field, CA: NASA.

Brudnicki, D.J., \& McFarland, A.L. (1997). User Request Evaluation Tool (URET) Conflict Probe Performance and Benefits Assessment. (MP97W112). McLean, VA: MITRE Corporation.

Canning, J.M., Johansson, J., Gronlund, S.D., Dougherty, M.R.P., \& Mills, S.H. (1999). Effects of a novel interface design on strategic planning by en route controllers. In R.S. Jensen and J.D. Callister (Eds.), Proceedings of the 10th International Symposium on Aviation Psychology (pp. 528533). Columbus, OH: Ohio State University Press.

David, H. (1997). Radical revision of en-route air traffic control, EEC Report No. 307, Eurocontrol Experimental Center, Brussells, Belgium.

Dougherty, M.R.P., Gronlund, S.D., Durso, F.T., Canning, J. \& Mills, S.H. (1999). Plan generation in air traffic control. In R. S. Jensen and J. D. Callister (Eds.), Proceedings of the 10th International Symposium on Aviation Psychology (pp. 534-539). Columbus, $\mathrm{OH}$ : Ohio State University Press.

Durso, F.D., \& Gronlund, S.D. (1999). Situation awareness. F.T. Durso, R. Nickerson, R. Schvaneveldt, S. Dumais, M. Chi, \& S. Lindsay (Eds.), The Handbook of Applied Cognition (pp. 283-314). New York: Wiley.

Hart, S.G., \& Staveland, L.E. (1998). Development of NASA-TLX (task load index): Results of empirical and theoretical research. In P.A. Nacock and N. Meshkati (Eds.), Human Mental Workload (pp. 139-181). Elsevier: North-Holland.

Hotelling, H. (1931). The generalization of Student's ratio. In:Annals of Mathematical Statistics, 2, 360-378.

Hutton, R.J.B., Olszewski, R., Thordsen, M.L., \& Kaempf, G.L. (1997). En route air traffic controller decision making and operational errors. Dayton, $\mathrm{OH}$ : Klein Associates. 
Marcucci, M. (1986). A comparison of the power of some tests for repeated measurements. Journal of Statistical Computation and Simulation, 26, 37-53.

Means, B., Mumaw, R.J., Roth, C., Schlager, M.S., McWilliams, E., Gagne', E., Rice, V., Rosenthal, D., \& Heon, S. (1988). ATC training analysis study: Design of the next-generation ATC training system. (Report No. FAA/OPM 342-036) Washington, DC: Department of Transportation/Federal Aviation Administration.

Meckoff, C., \& Gibbs, P. (1994). PHARE Highly Interactive Problem Solver, Eurocontrol report 273, Brussels, Belgium.

Miller, T.E., Copeland, R., Heaton, J.K., \& McCloskey, M.F. (1998). A cognitive approach to developing decision support tools for air campaign planners. (Technical report prepared under contract F3060295-C-0216 for Rome Laboratory) Fairborn, OH: Klein Associates Inc.
Moertl, P.M., Canning, J.M., Johansson, J., Gronlund, S.D., Dougherty, M.R.P., \& Mills, S.H. (2000). Workload and Performance in FOPA: A Strategic Planning Interface for Air Traffic Control. Proceedings of the 44th annual meeting of the Human Factors and Ergonomics Society, 3.65 - 3.68.

Pew, R.W., \& Mavor, A.S. (1998). Panel on Modeling Human and Organizational Behavior: Applications to Military Simulation. Washington, DC: National Academy Press.

Taylor, R.M., Finnie, S., \& Hoy, C. (1997). Cognitive rigidity: The effects of mission planning and automation on cognitive control in dynamic situations. Paper presented at the 9th International Symposium on Aviation Psychology, Columbus, $\mathrm{OH}$.

Vivona, R.A., Ballin, M.G., Green, S.M., Bach, R.E, \& McNally, B.D. (1996). A system concept for facilitating user preferences in en route airspace, NASA Technical Memorandum 4763, Ames Research Center, Moffett Field, CA.

Xiao, Y., Milgram, P., \& Doyle, D. J. (1997). Capturing and modeling planning expertise in anesthesiology: A field study. In C. E. Zsambok \& G. Klein's (Eds.) Naturalistic Decision Making, p.p. 197 207. Mahwah, NJ: Erlbaum. 